\title{
Facteurs D'adoption de la Gestion Intégrée des Ravageurs en Production Maraîchère Dans la Vallée du Niger au Bénin
}

\section{Claude Codjo Kpadenou,}

Laboratoire d'Analyse et de Recherches sur les Dynamiques Economiques et Sociales, Faculté d'Agronomie, Université de Parakou, République du Bénin

\section{Clarisse Tama,}

Département de Sociologie, Faculté des Lettres, Arts et Sciences Humaines, Faculté d'Agronomie, Université de Parakou, République du Bénin

\section{Baké Dado Tossou,}

Laboratoire Société-Environnement (laSEn), Faculté d'Agronomie (FA),

Université de Parakou (UP), République du Bénin

Jacob Afouda Yabi,

Laboratoire d'Analyse et de Recherches sur les Dynamiques Economiques et Sociales, Faculté d'Agronomie, Université de Parakou, République du Bénin

\section{Résume}

Les pertes de récoltes dues aux insectes nuisibles, maladies, nématodes et mauvaises herbes, en production maraîchère, restent importantes au Bénin. Les solutions proposées pour combattre ces insectes nuisibles sont axées sur la lutte chimique. Compte tenu de la sécurité sanitaire, la qualité nutritionnelle et le respect de l'environnement, la recherche de luttes alternatives s'impose. La présente étude a pour objectif d'identifier les facteurs qui favorisent ou limitent l'adoption de la gestion intégrée des ravageurs (GIR) dans le secteur maraîcher dans la vallée du Niger au Bénin. Pour ce faire, une enquête a été conduite auprès de 280 maraîchers échantillonnés dans sept villages de la vallée de manière aléatoire. Des données relatives aux caractéristiques socioéconomiques des maraîchers et les pratiques de GIR utilisées dans la production maraîchère ont été collectées. Le modèle de régression Logit a été utilisé pour la détermination des facteurs affectant l'adoption des pratiques de GIR. Les résultats montrent que ce sont les pratiques de lutte culturale qui sont plus adoptées par les maraîchers enquêtés pour la GIR. Par contre, les pratiques biologiques et physiques sont moins adoptées pour le contrôle des bioagresseurs. Par ailleurs, la superficie emblavée, l'expérience dans le maraîchage, l'éducation, la résistance des ravageurs, le contact avec une ONG 
ou un projet et l'appartenance à un groupement constituent les déterminants majeurs de l'adoption des pratiques de la GIR. Au vu de ces résultats, la promotion des pratiques GIR en vue d'augmenter leur niveau d'adoption, passe indubitablement par un suivi rapproché et une formation des maraîchers.

Mots clés : Maraîchage, GIR, Adoption, Logit, Vallée du Niger, Bénin

\section{Factors for the Adoption of Integrated Pest Management in Vegetable Production in the Niger Valley in Benin}

\section{Claude Codjo Kpadenou,}

Laboratoire d'Analyse et de Recherches sur les Dynamiques Economiques et Sociales, Faculté d'Agronomie, Université de Parakou, République du Bénin

\section{Clarisse Tama,}

Département de Sociologie, Faculté des Lettres, Arts et Sciences Humaines, Faculté d'Agronomie, Université de Parakou, République du Bénin

\section{Baké Dado Tossou,}

Laboratoire Société-Environnement (laSEn), Faculté d'Agronomie (FA),

Université de Parakou (UP), République du Bénin

\section{Jacob Afouda Yabi,}

Laboratoire d'Analyse et de Recherches sur les Dynamiques Economiques et Sociales, Faculté d'Agronomie, Université de Parakou, République du Bénin

\section{Abstract}

Harvest losses due to harmful insects, diseases, nematodes and weeds, in vegetable production, remain significant in Benin. The solutions proposed to combat these harmful insects are focused on chemical control. Given health security, nutritional quality and respect for the environment, the search for alternative struggles is essential. This study aims to identify the factors that favor or limit the adoption of Integrated Pest Management (IPM) in the vegetable sector in the Niger Valley in Benin. To do this, a survey was conducted among 280 market gardeners randomly sampled in seven villages in the valley. Based on an individual questionnaire, data on the socioeconomic characteristics of market gardeners and the IPM practices used in vegetable production were collected. The Logit regression model was used to identify factors affecting the adoption of IPM practices. The results show that 
the cultural control practices are more adopted by market gardeners investigated for the IPM. On the other hand, biological and physical practices are less adopted for the control of pests. In addition, the area planted, the experience in market gardening, education, pest resistance, contact with an NGO or a project and membership of a group are the determining factors of the adoption of the practices of the IPM. In view of these results, the promotion of IPM practices in order to increase their level of adoption undoubtedly involves close monitoring and training of market gardeners.

Keywords: Market gardening, IPM, Adoption, Logit, Niger Valley, Benin

\section{Introduction}

Au Bénin, la vallée du Niger constitue l'une des grandes zones productrices des cultures maraîchères. Elles sont constituées d'une part, des cultures traditionnelles de plein champ telles que la tomate, le piment, l'oignon, le gombo et les légumes feuilles et d'autre part, des cultures exotiques à savoir la carotte, le chou, la laitue, le concombre, le poireau, le haricot vert, etc. (Biao et al.,2016; Tiamyhou et al.,2003). Outre son importance alimentaire et nutritionnelle, le maraîchage représente aujourd'hui la principale activité pour plusieurs ménages qui s'y adonnent, pour lesquels il constitue une source importante de revenu financier. Mais malheureusement, cette activité reste sujette à des contraintes dont l'une des plus importantes est les attaques d'insectes et des pathologies (Adékambi et al., 2008). Ainsi, dans le but de limiter la perte de récoltes due aux dégâts causés par les insectes nuisibles, les maladies, les nématodes et les mauvaises herbes afin de maximiser leur profit, les maraîchers sont contraints d'utiliser de façon anarchique et abusive les pesticides chimiques. Cette forte utilisation inappropriée des pesticides chimiques, hautement toxiques, engendre des conséquences négatives sur l'environnement, la santé des humains et des animaux, et la résistance des bioagresseurs (Son et al., 2018 ; AssogbaKomlan et al., 2007).

Face à cette problématique, les concepts de méthodes alternatives de lutte et de Gestion Intégrée des Ravageurs (GIR) a été promue et testée par les différents projets et programmes de vulgarisation afin de réduire ces effets néfastes des pesticides chimiques de synthèse (Houndété et al., 2010; Fanou et al., 2004 ). En effet, la GIR est un système de lutte conçu qui, compte tenu $\mathrm{du}$ milieu particulier et de la dynamique des populations des espèces considérées, utilise toutes les techniques et méthodes appropriées de façon aussi compatible que possible en vue de maintenir les populations d'organismes nuisibles à des niveaux où ils ne causent pas de dommages économiques (FAO,2011). Elle associe les méthodes de luttes agronomique ou culturale, variétale/génétique, biologique et chimique plus raisonnées. 
Malgré toutes les performances démontrées des pratiques de la GIR reconnues des maraîchers eux-mêmes, leur adoption reste encore limitée (Adékambi et al., 2010). Ainsi, quels sont les facteurs qui favorisent ou limitent l'adoption de la lutte intégrée dans le secteur maraîcher de la vallée du Niger au Bénin? Afin d'y répondre, la présente étude se fixe pour objectifs d'identifier les facteurs susceptibles d'influencer la probabilité d'adoption de cette technologie.

\section{Matériels et méthodes}

\subsection{Matériels}

\section{Zone d'étude}

La Vallée du Niger (côté Bénin) est située à l'extrême Nord de la République du Bénin. Elle s'étale sur les Communes de Malanville et de Karimama se trouvant entre $11^{\circ} 30$ et $12^{\circ} 30$ de latitude Nord d'une part et les méridiens $2^{\circ} 43$ et $3^{\circ} 20$ de longitude Est d'autre part. Ces communes sont limitées au Nord par le fleuve Niger, au Sud par les Communes de Banikoara, Kandi et Ségbanan, à l'Est par le fleuve Niger et la République Fédérale du Nigeria et à l'Ouest par le Burkina Faso (Figure 1).

\section{VALLEE DU NIGER DU COTE DU BENIN}

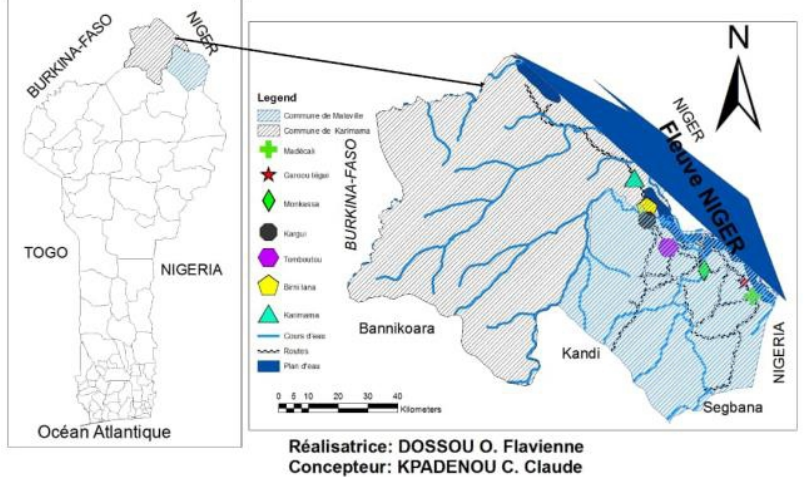

Deux principaux critères ont servi de guide dans le choix des villages d'étude : l'importance du maraîchage et l'accessibilité des villages. Il s'agit de: Toumboutou, Garou-Tédji, Madécali et Monkassa (dans la commune de Malanville), Kargui, Birni-Lafia et Kotchi (dans la commune de Karimama). Cette inégale répartition est due au fait que la commune de Malanville constitue la grande zone de production maraîchère dans la Vallée du Niger.

\section{Echantillonnage et collecte des données}

L'unité d'observation de la recherche est le chef de ménage. La taille minimale de l'échantillon a été obtenue en utilisant l'approximation normale de la distribution binomiale proposée par (Dagnelie, 1998 ) : $\mathrm{N}=\left[\left(\mathrm{U}_{1-\alpha / 2}\right)^{2} \mathrm{x}\right.$ 
$\mathrm{p}(1-\mathrm{p})] / \mathrm{d}^{2}$, avec $\mathrm{U}_{1-\alpha / 2}$ la valeur de la variable aléatoire normale pour la valeur de probabilité de $1-\alpha / 2$, $\alpha$ étant le risque d'erreur. Pour $\alpha=5 \%$, la probabilité de $1-\alpha / 2$ est égale à 0,975 et on a $U_{1-\alpha / 2}=1,96$. p est la proportion de personnes qui s'adonnent à la production de cultures de contre-saison dans le milieu d'étude et d la marge d'erreur d'estimation, retenue à $5 \%$ dans cette étude.

A partir des valeurs de $\mathrm{p}=0.24$ issues des résultats de la phase exploratoire de l'étude, au 1total deux cent quatre-vingts (280) maraîchers ont été sélectionnés, soit quarante (40) enquêtés par village. Dans chaque localité, les personnes enquêtées ont été identifiées selon un échantillonnage aléatoire simple.

Sur la base d'un questionnaire rempli à travers des enquêtes individuelles et des focus groupes, des données primaires ont été collectées. Ces données sont relatives aux caractéristiques socio-économiques des maraîchers et aux pratiques de luttes intégrées utilisées dans la production.

Dans le cadre de la présente étude, seules les pratiques de la GIR les plus vulgarisées au Bénin ont été considérées. Il s'agit :

- pour la lutte biologique : utilisation des extraits aqueux de plantes et de biopesticides ;

- pour la lutte culturale : utilisation de variétés tolérantes/résistantes, la rotation culturale, les associations culturales (avec des plantes répulsives ou attractives);

- pour la lutte physique/ mécanique : arrachage et brûlage des plantes infectées, couverture de pépinière avec des filets ;

- pour la lutte chimique : utilisation des pesticides de synthèse.

\subsection{Méthode d'analyse}

\section{Cadre théoriques}

Dans le domaine agricole, l'innovation est comprise comme l'introduction d'une pratique agricole nouvelle ou la modification d'une pratique traditionnelle, et dans une moindre mesure comme l'adoption d'un nouveau comportement socioéconomique meilleur et plus bénéfique (Petit, 2015). La gestion intégrée des nuisibles des cultures fait appel à une combinaison judicieuse de toutes les pratiques ou méthodes de lutte disponibles. Elle n'est donc pas une simple technologie ou innovation que les producteurs peuvent adopter en un coup (Bourgeault, 2009 ; James et al., 2010). Cependant, les différentes technologies ou pratiques de lutte entrant dans le cadre de la gestion intégrée sont adoptées par les producteurs en fonction des avantages comparatifs. Selon les théories économiques, une technologie ou innovation n'est adoptée que lorsque les individus concernés sont convaincus par l'intérêt ou le gain qu'ils peuvent en tirer (CIMMYT, 1993). Ainsi, en nous rapportant à l'objectif du producteur qui selon Rasmussen (2011) est d'optimiser, voire de maximiser son profit sous 
contrainte des coûts de production, il est possible d'émettre l'hypothèse selon laquelle «l'adoption d'une technologie est fonction de facteurs économiques liés au producteurs ». Les rapports sociaux influençant les choix opérés par le producteur (CIRAD-GRET, 2002), l'hypothèse précédente apparait incomplète. Tenant donc compte de l'environnement de l'exploitation agricole (Relations avec d'autres agents économiques: agriculteurs voisins, propriétaires fonciers, commerçants, agents de développement rural, etc.), l'adoption d'une technologie agricole par le producteur est fonction de facteurs socio- démographiques et économiques qui lui sont est liés. En se référant aux fondements théoriques ci-dessus et des résultats de plusieurs études antérieures sur l'adoption de technologie dans le domaine agricole, notamment ceux de Hasan,(2017); Odjouola,(2017); Yabi et al., (2016); Zongo et al.(2015) ; Parsa et al. (2014) ; Adétonah et al., (2011) et Adekambi et al., (2010), il est possible de supposer que la probabilité de l'adoption de la gestion intégrés des ravageurs par les maraîchers de la vallée du Niger comme une fonction de certaines variables exogènes sociodémographiques et économique.

\section{Modèles empirique et conceptuel}

Dans le cadre de cette étude, nous avons retenu le modèle Logit, souvent utilisé dans les études d'adoption des technologies pour des raisons de commodité (Cimmyt, 1993). En effet, les modèles Logit polytomiques non ordonnés sont moins intensifs en calculs mais requièrent la non violation de l'hypothèse de l'indépendance des alternatives non pertinentes (Madalla, 1985; Cramer, 1991). Quant aux modèles Probit polytomiques non ordonnés, ils sont plus intensifs en calculs et n'imposent pas de restrictions particulières (Sale et al., 2014).

La décision d'adopter une innovation n'intervient que lorsque l'effet combiné des facteurs atteint une valeur à partir de laquelle le décideur accepte d'utiliser ou adopter l'innovation. En se mettant dans l'hypothèse que l'effet est mesuré par un indice non observable $I_{d}$ pour le décideur $d$ et $I_{0 d}$ la valeur critique de l'indice à partir de laquelle il adopte la technologie, deux cas de figures peuvent se présenter :

-Si $\mathrm{I}_{\mathrm{d}}$ est supérieur ou égal à $\mathrm{I}_{0 \mathrm{~d}}$, alors il adopte la technologie et la variable d'adoption $Y$ prend la valeur 1 . Plus l'indice $I_{d}$ est supérieur à la valeur critique, plus la probabilité pour que le producteur adopte est élevée. Si $\mathrm{I}_{\mathrm{d}}$ est inférieur à $\mathrm{I}_{0 \mathrm{~d}}$, il rejette l'innovation et $\mathrm{Y}$ est égal à 0 .

En formulation mathématique, il vient que :

$$
\left\{\begin{array}{l}
I_{d} \geq I_{0 d} \Rightarrow \mathrm{Y}=1 \\
I_{d}<I_{0 d} \Rightarrow \mathrm{Y}=0
\end{array}\right.
$$


-Pour l'individu d, l'indice $\mathrm{I}_{\mathrm{d}}$ peut être une combinaison linéaire de variables $\mathrm{Xi}$ qui déterminent l'adoption et de coefficients $\beta \mathrm{i}$ à estimer. Son expression est alors mathématiquement donnée par :

$$
I_{d}=\sum_{i=1}^{k} \beta_{i} X_{i d}
$$

Avec $X_{\text {id }}$ la iième variable indépendante expliquant l'adoption de la technologie par l'individu $\mathrm{d}$ et $\beta$ i son paramètre correspondant à estimer.

La probabilité $\mathrm{P}_{\mathrm{d}}$ pour que l'individu d adopte l'innovation est alors :

$$
\mathrm{Pd}=\mathrm{P}(\mathrm{Y}=1)
$$

Comme l'indice $\mathrm{I}_{0 \mathrm{~d}}$ est une variable aléatoire, si nous désignons par $\mathrm{F}$ sa fonction de probabilité cumulée ou fonction de répartition, il vient que :

$$
\left\{\begin{array}{l}
P(Y=1)=P\left(I_{0 d} \leq I_{d}=F\left(I_{d}\right)\right. \\
P(Y=0)=P\left(1-F\left(I_{d}\right)\right.
\end{array}\right.
$$

La forme fonctionnelle de $\mathrm{F}$ est déterminée par celle de la fonction de densité de probabilité de la variable aléatoire Id. Pour le modèle logit, il s'agit d'une fonction logistique de la forme :

$$
F(x)=\frac{1}{1+e^{-X}}=\frac{1}{1+e^{-\left(\beta_{0}+\beta_{i} Z\right)}}
$$

\section{Modèle économétrique}

L'équation empirique issue du modèle théorique, se présente comme suit :

$$
\begin{gathered}
P\left(Y_{i}=1 / A D O P\right)=\frac{1}{1+e^{-X}} \\
\text { Avec } X=\beta_{0}+\beta_{1} M A L A N+\beta_{2} S E X+\beta_{3} \text { ACTPRIN }+\beta_{4} E X P+\beta_{5} S U P+ \\
\beta_{6} \text { ACTIF }+\beta_{7} E D U C F+\beta_{8} E D U C I+\beta_{9} \text { ACTPRIN }+\beta_{10} M F V D+ \\
\beta_{11} \text { GROUP }+\beta_{12} \text { ONG }+\beta_{13} \text { VULG }+\beta_{14} C R E D+e_{1}
\end{gathered}
$$

Où $\beta_{0}$ est le terme constant; $\beta_{i}$ les coefficients à estimer, et ei les termes d'erreur. Plusieurs techniques sont utilisées pour juger de la qualité du modèle : elle peut être donnée par la vraisemblance du modèle qui suit une loi de Chideux. Le modèle est dit globalement bon, lorsque la valeur de la vraisemblance est supérieure à celle du Chi deux au même degré de liberté à un seuil donné $(1 \%, 5 \%$ ou $10 \%)$, ou directement lorsque la probabilité de LR est inférieure au seuil de signification choisi. Les signes indiquent dans quel sens la variation de la variable explicative influe sur la variation de la variable expliquée. A chaque signe des coefficients, est associée une signification qui revêt une grande importance. Leur signification est donnée par une probabilité qui indique dans quel intervalle de confiance on peut compter sur le signe. Toutes les variables explicatives introduites dans le modèle sont décrites dans le Tableau 1. Les logiciels SPSS et STATA ont été utilisés respectivement pour les statistiques descriptives et les régressions économétriques. 
Tableau 1 : Description et statistiques descriptives des variables incluses dans les modèles Logit

\begin{tabular}{|c|c|c|c|c|c|c|}
\hline \multirow[b]{2}{*}{ Variables } & \multirow[b]{2}{*}{ Code } & \multicolumn{2}{|c|}{ Variables quantitatives } & \multicolumn{2}{|c|}{ Variables qualitatives } & \multirow{2}{*}{$\begin{array}{l}\text { Signes } \\
\text { attendus }\end{array}$} \\
\hline & & Moyenne & $\begin{array}{l}\text { Ecart- } \\
\text { type }\end{array}$ & Oui $=1$ & Non $=0$ & \\
\hline Superficie cultivée & SUP & 2,08 & 1,65 & & & \pm \\
\hline Expérience & EXP & 17,25 & 7,48 & & & + \\
\hline Actif agricole & ACTIF & 5,08 & 2,87 & & & + \\
\hline $\begin{array}{l}\text { Commune } \\
\text { Malanville }\end{array}$ & MALAN & & & $160(54,14)$ & $120(42,86)$ & \pm \\
\hline Sexe masculin & SEXE & & & $259(92,5)$ & $21(7,5)$ & \pm \\
\hline $\begin{array}{l}\text { Maraîchage comme } \\
\text { activité principale }\end{array}$ & $\begin{array}{l}\text { ACTPRI } \\
\mathrm{N}\end{array}$ & & & $208(74,29)$ & $72(25,71)$ & + \\
\hline $\begin{array}{l}\text { Mode de faire valoir } \\
\text { direct }\end{array}$ & MFVD & & & $213(75,90)$ & $67(45,86)$ & + \\
\hline Education formelle & EDUCF & & & $92(32,86)$ & $188(67,14)$ & + \\
\hline $\begin{array}{l}\text { Education } \\
\text { informelle }\end{array}$ & EDUCIN & & & $44(15,71)$ & $236(84,29)$ & + \\
\hline $\begin{array}{l}\text { Accès à la } \\
\text { vulgarisation }\end{array}$ & VULG & & & $220(78,57)$ & $60(21,43)$ & + \\
\hline $\begin{array}{l}\text { Contacts avec ONG } \\
\text { ou projet }\end{array}$ & ONG & & & $214(76,43)$ & $66(23,57)$ & + \\
\hline $\begin{array}{l}\text { Appartenance à un } \\
\text { groupement }\end{array}$ & GROUP & & & $182(65)$ & $98(35)$ & + \\
\hline $\begin{array}{l}\text { Accès au crédit } \\
\text { agricole }\end{array}$ & CRED & & & $73(26,07)$ & $207(73,93)$ & + \\
\hline
\end{tabular}

\section{Résultats}

\subsection{Caractéristiques socioéconomiques des maraîchers}

Les unités enquêtées au cours de cette étude sont constituées aussi bien d'hommes que de femmes. Les chefs d'exploitation sont en général des hommes $(92,5 \%)$ sauf des cas rares où la femme représente le chef du ménage $(7,5 \%)$ par suite du décès de son époux ou d'un divorce. Le maraîchage demeure la principale occupation de $74,29 \%$ des enquêtés qui enregistrent une expérience moyenne de 17,25 \pm 7,48ans dans le maraîchage. Les taux de scolarisation $(32,86 \%)$ et d'alphabétisation $(15,71 \%)$ sont faibles. Les observations sur la taille des ménages de la zone d'étude montrent qu'ils sont pour la plupart de grande taille du fait que les pratiques traditionnelles de communautarisme y sont encore plus fortes. Ces ménages comptent en moyenne $12 \pm 5$ personnes dont $5 \pm 3$ actifs agricoles. Les producteurs enquêtés ont acquis une certaine facilité à se mettre en groupement et de bénéficier de l'appui des agents d'encadrement. Ainsi, 65\% des maraîchers appartiennent à des groupements ou associations de maraîchers. En matière de contrôle des ravageurs, l'utilisation de pesticides chimiques constitue la pratique la plus courante $(91,43 \%$ des enquêtés). Seules $8,57 \%$ des 
producteurs utilisent uniquement des biopesticides. Concernant l'encadrement technique des producteurs, 73,93\% parmi eux bénéficient des appuis des agents de l'Agence Territoriale du Développement Agricole (ATDA). Par ailleurs, 59,64\% des enquêtés ont reçu une formation en GIR. Ces tendances montrent qu'un effort important, en faveur de la filière maraîchère, reste à fournir pour changer le comportement des producteurs en matière d'utilisation des pesticides chimiques. Les enquêtés emblavent en moyenne 2,08 \pm 1,66 ha. L'accès au crédit parait assez problématique pour les producteurs de cultures de contre-saison dans la vallée du Niger. Ainsi, seulement 32,07\% des producteurs enquêtés ont accès au crédit agricole et obtiennent en moyenne auprès des microfinances 177489,29 \pm 402836,64 FCFA.

\subsection{Adoption des pratiques de gestion intégrée des ravageurs}

Le Tableau 2 illustre le taux d'adoption de la GIR. Dans cette étude, sept pratiques de GIR ont été considérées et regroupées en trois catégories. Dans l'ensemble ce sont les pratiques de lutte culturale qui sont plus utilisées par les producteurs enquêtés pour la GIR. Ainsi, 86,79\% des enquêtés utilisent des semences de variétés tolérantes ou résistantes et $89,64 \%$ font de la rotation culturale.

Tableau 2 : Répartition des maraîchers selon des pratiques de la GIR

\begin{tabular}{|c|c|c|c|c|c|c|}
\hline & \multicolumn{2}{|l|}{ Malanville } & \multicolumn{2}{|l|}{ Karimama } & \multicolumn{2}{|l|}{ Echantillon } \\
\hline & $\begin{array}{l}\text { Fréquence } \\
\text { absolue }\end{array}$ & $\begin{array}{l}\text { Fréquence } \\
\text { relative }\end{array}$ & $\begin{array}{l}\text { Fréquence } \\
\text { absolue }\end{array}$ & $\begin{array}{l}\text { Fréquence } \\
\text { relative }\end{array}$ & $\begin{array}{l}\text { Fréquence } \\
\text { absolue }\end{array}$ & $\begin{array}{l}\text { Fréquence } \\
\text { relative }\end{array}$ \\
\hline \multicolumn{7}{|c|}{ Luttes biologiques } \\
\hline $\begin{array}{ll}\text { Extraits } & \text { de } \\
\text { feuilles } & \text { de } \\
\text { plantes } & \end{array}$ & 28 & 17,5 & 19 & 15,83 & 47 & 16,79 \\
\hline Biopesticides & 12 & 7,5 & 7 & 5,83 & 19 & 6,79 \\
\hline \multicolumn{7}{|c|}{ Luttes culturales } \\
\hline $\begin{array}{l}\text { Variétés } \\
\text { tolérantes ou } \\
\text { résistantes }\end{array}$ & 132 & 82,5 & 111 & 92,5 & 243 & 86,79 \\
\hline $\begin{array}{l}\text { Rotation } \\
\text { culturale } \\
\text { Associations } \\
\text { culturales }\end{array}$ & 138 & 86,25 & 113 & 94,17 & 251 & 89,64 \\
\hline \multicolumn{7}{|c|}{ Luttes physiques ou mécaniques } \\
\hline $\begin{array}{l}\text { Arrachage et } \\
\text { brûlage des } \\
\text { plantes } \\
\text { malades }\end{array}$ & 27 & 16,88 & 12 & 10 & 39 & 13,93 \\
\hline $\begin{array}{l}\text { Couverture de } \\
\text { pépinière avec } \\
\text { des filets }\end{array}$ & 58 & 36,25 & 40 & 33,33 & 98 & 35 \\
\hline
\end{tabular}




\subsection{Facteurs déterminants de la gestion intégrée des ravageurs}

L'analyse du Tableau 3 révèle que les modèles d'estimation des facteurs d'adoption des pratiques de lutte biologique, de lutte agronomique sont significatifs au seuil de 1\%. Quant au modèle d'estimation des facteurs d'adoption de lutte physique, il est significatif au seuil de 5\%. L'estimation des modèles donne respectivement un pseudo- $\mathrm{R}^{2}$ de McFadden de 0,429 ; 0,392 et 0,172 pour les modèles d'adoption des pratiques de lutte biologique, de lutte agronomique et de lutte physique. Les variations des variables explicatives incluses dans le modèle expliquent donc respectivement à 42,9\%, $39,2 \%$, et $17,2 \%$ les variations de l'adoption des pratiques de lutte biologique, de lutte agronomique et de lutte physique.

\section{Facteurs déterminant l'adoption des pratiques de lutte biologique de GIR}

Les résultats du modèle d'estimation des facteurs d'adoption des pratiques de lutte biologique de la GIR montrent que les variables telles que la superficie emblavée en maraîchage, le nombre d'actifs agricoles, la résistance des ravageurs et le contact avec une ONG ou un projet sont significatives au seuil de $5 \%$; le nombre d'années d'expérience dans le maraîchage et l'éducation formelle sont significatives au seuil de 10\% (tableau 4).

La superficie emblavée : elle a une influence négative sur la probabilité du choix de luttes biologiques comme pratique de gestion intégrée des ravageurs. Cependant, il est à relever qu'avec le signe négatif du coefficient, ce résultat nous indique que plus la superficie emblavée est grande, moins le maraîcher aura tendance à adopter cette stratégie face aux ravageurs des cultures.

Le nombre d'actifs agricoles : il a une influence positive sur l'adoption de luttes biologiques comme pratique de la GIR. Cela suppose que, plus la main-d'œuvre familiale est disponible dans le ménage de l'enquêté, plus la probabilité qu'il choisisse cette pratique est élevée.

L'éducation formelle: il affecte positivement l'adoption de lutte biologique comme pratique de gestion intégrée des ravageurs. Ainsi, les maraîchers instruits formellement ont tendance à plus adopter de lutte biologique que les maraîchers non instruits.

La résistance aux ravageurs: elle est une variable qui influence négativement l'adoption de lutte biologique comme pratique de la GIR. Ce résultat traduit que les maraîchers qui font face à des ravageurs résistants utilisent moins les pratiques biologiques.

Le contact avec une ONG ou un projet : il influence positivement les luttes biologiques comme pratique de gestion intégrée des ravageurs. Cela suppose que les maraîchers ayant eu un contact avec une ONG ou un projet adoptent mieux les pratiques de lutte biologique. 


\section{Facteurs déterminant l'adoption des pratiques de lutte agronomiques de la GIR}

L’analyse des résultats du Tableau 5 montre que les pratiques de culturale de la GIR sont diversement influencées par les variables considérées.

La superficie emblavée pour le maraîchage est significative au seuil de $1 \%$ et influence positivement l'utilisation de la lutte culturale comme méthode de la GIR. Cela suppose que les maraîchers qui emblavent de grandes superficies optent pour l'utilisation des pratiques culturales de la GIR.

$L$ 'expérience dans le maraîchage a un impact significatif et positif au seuil de $1 \%$ sur l'adoption de la lutte culturale. Plus le maraîcher est expérimenté, plus il songe à l'utilisation de la pratique de lutte culturale comme la GIR. L'âge étant proxy de l'expérience, les jeunes sont donc moins disposés à l'utilisation de cette pratique que les personnes âgées.

Le nombre d'actifs agricoles a une influence positive et significative au seuil de 1\% sur l'adoption de la lutte culturale comme méthode de la GIR. Cela suppose que, plus la main-d'œuvre familiale est disponible dans la zone, plus la probabilité de choisir la lutte culturale est élevée.

L'éducation formelle affecte positivement de manière significative au seuil 1\% l'adoption de lutte culturale comme pratique de la GIR. Ainsi, les maraîchers instruits formellement ont tendance à plus adopter de la lutte culturale que les maraîchers non instruits.

La résistance aux ravageurs est une variable qui influence positivement l'adoption de lutte culturale comme pratiques de gestion intégrée des ravageurs. Ce résultat traduit que les maraîchers qui font face à des ravageurs résistants adoptent plus les pratiques culturales de la GIR.

L'appartenance à un groupement est significative au seuil de $10 \%$ et influence négativement la probabilité que le producteur adopte la lutte agronomique comme pratique de la GIR. Ce résultat traduit que, plus le producteur appartient au groupement, plus il opte pour l'utilisation de la pratique culturale de la GIR.

\section{Facteurs déterminant l'adoption des pratiques de lutte physique ou mécanique de la GIR}

Les résultats du modèle logistique identifient trois facteurs déterminants l'adoption luttes physiques ou mécaniques (Tableau 6).

La superficie emblavée pour le maraîchage : elle a un impact négatif et significatif au seuil de $10 \%$ sur l'utilisation des pratiques de lutte physique ou mécanique de la GIR. Cela suppose que les maraîchers qui emblavent de grandes superficies optent moins pour l'utilisation des pratiques physiques de la GIR.

Le sexe du producteur est significatif au seuil de $10 \%$ et influence négativement l'utilisation de la lutte physique comme méthode de la GIR. Cela 
suppose que les hommes utilisent plus la pratique de lutte physique que les femmes.

Le nombre d'actifs agricoles: il a une influence positive et significative au seuil de $1 \%$ sur l'adoption de lutte physique comme pratique de la GIR. Cela suppose que, plus la main-d'œuvre familiale est disponible dans le ménage de l'enquêté, plus la probabilité de qu'il choisisse cette pratique est élevée.

Tableau 3 : Résumés des modèles d'estimation des facteurs d'adoption.

\begin{tabular}{cccc}
\hline & Luttes biologiques & Luttes culturales & $\begin{array}{c}\text { Luttes physiques ou } \\
\text { mécaniques }\end{array}$ \\
\hline $\begin{array}{c}\text { Nombre } \\
\text { d'observation }\end{array}$ & 280 & 280 & 280 \\
Log pseudo & 117,275 & 124,389 & 159,348 \\
likelihood & $0,429 * * *$ & $0,392 * * *$ & $0,172 *$ \\
Pseudo R & 0,000 & 0,000 & 0,051 \\
Probabilité de & & & \\
signification & $* * *$ significatif à $1 \% ; * *$ significatif à $5 \% ; *$ significatif à $10 \%$
\end{tabular}

Tableau 4 : Résultat du modèle d'estimation des facteurs d'adoption de lutte biologique

\begin{tabular}{|c|c|c|c|}
\hline Variables & Coefficients & Erreurs standard & Signification \\
\hline Commune & $-0,262$ & 0,625 & 0,670 \\
\hline Sexe & 0,418 & 0,620 & 0,501 \\
\hline $\begin{array}{l}\text { Maraîchage comme activité } \\
\text { principale }\end{array}$ & 0,0978 & 1,714 & 0,954 \\
\hline Expérience en maraîchage & $-0,983^{*}$ & 0,146 & 0,062 \\
\hline Superficie emblavée & $-0,196 * *$ & 0,592 & 0,073 \\
\hline Actifs agricoles & $1,407 * *$ & 0,586 & 0,016 \\
\hline Education formelle & $0,915^{*}$ & 0,617 & 0,031 \\
\hline Education informelle & $-0,528$ & 0,656 & 0,421 \\
\hline Mode de faire valoir & 0,297 & 0,577 & 0,606 \\
\hline Résistance des ravageurs & $-1,758 * *$ & 0,553 & 0,002 \\
\hline Accès à la vulgarisation & 0,158 & 0,303 & 0,601 \\
\hline Contacts avec ONG ou projet & $1,973 * *$ & 0,636 & 0,002 \\
\hline Appartenance à un groupement & 0,111 & 0,475 & 0,815 \\
\hline Accès au crédit agricole & $-0,383$ & 0,620 & 0,537 \\
\hline
\end{tabular}

***significatif à $1 \% ; * *$ significatif à $5 \%$; *significatif à $10 \%$

Tableau 5: Résultat du modèle d'estimation des facteurs de l'adoption de lutte culturale

\begin{tabular}{llll}
\hline Variables & Coefficients & Erreurs standard & Signification \\
\hline Commune & 0,059 & 0,448 & 0,895 \\
Sexe & 0,419 & 0,599 & 0,485 \\
Maraîchage comme activité & 0,929 & 1,885 & 0,622 \\
principale & & & \\
Expérience en maraîchage & $1,824 * * *$ & 0,559 & 0,008 \\
Superficie emblavée & $2,720 * * *$ & 0,647 & 0,000 \\
Actifs agricoles & $0.113 * * *$ & 0.035 & 0,001 \\
Education formelle & $1,383 * * *$ & 0,366 & 0,000 \\
\hline
\end{tabular}




\begin{tabular}{llll}
\hline Education informelle & 0,00 & 0,026 & 0,992 \\
Mode de faire valoir & 0,088 & 0,163 & 0,586 \\
Résistance des ravageurs & $1,824^{* *}$ & 0,558 & 0,008 \\
Accès à la vulgarisation & 0,055 & 0,441 & 0,892 \\
Contacts avec ONG ou projet & $-0,02$ & 0,03 & 0,543 \\
Appartenance à un groupement & $0,088^{*}$ & 0,053 & 0,096 \\
& & & 0,670 \\
Accès au crédit agricole & $-0,262$ & 0,625 & $0.6 \%$ \\
\hline
\end{tabular}

***significatif à $1 \% ; * *$ significatif à $5 \%$; *significatif à $10 \%$

Tableau 6: Résultat du modèle d'estimation des facteurs d'adoption de luttes physiques ou mécaniques

\begin{tabular}{lccc}
\hline Variables & Coefficients & Erreurs standard & Signification \\
\hline Commune & 0,297 & 0,578 & 0,610 \\
Sexe & $1,825^{*}$ & 0,979 & 0,062 \\
$\begin{array}{l}\text { Maraîchage comme } \\
\text { principale }\end{array}$ & 0,220 & 0,569 & 0,699 \\
$\begin{array}{l}\text { Expérience en maraîchage } \\
\text { Superficie emblavée }\end{array}$ & 0,469 & 0,589 & \\
Actifs agricoles & $-0,392^{*}$ & 0,233 & 0,425 \\
Education formelle & 2,438 & 0,588 & 0,093 \\
Education informelle & 0,00 & 0,085 & 0,000 \\
Mode de faire valoir & 0,045 & 0,023 & 0,975 \\
Résistance des ravageurs & 0,024 & 0,024 & 0,110 \\
Accès à la vulgarisation & 0,098 & 1,714 & 0,318 \\
Contacts avec ONG ou projet & $-0,528$ & 0,656 & 0,954 \\
Appartenance à un groupement & 0,357 & 0,661 & 0,421 \\
Accès au crédit agricole & 0,100 & 0,065 & 0,589 \\
\hline
\end{tabular}

***significatif à $1 \% ; * *$ significatif à $5 \% ; *$ significatif à $10 \%$

\section{Discussion}

Dans la zone d'étude le maraîchage est conduit par une population à dominance masculine. Cette situation n'est pas limitée au seul cas du maraîchage, mais s'observe dans l'agriculture en général. Cela pourrait s'expliquer par le fait que le maraîchage est une activité destinée au marché dans la vallée du Niger, c'est-à-dire une activité qui offre un revenu monétaire, la forte représentation des hommes est donc justifiée, car en Afrique, les hommes sont plus orientés vers les cultures qui offrent un revenu monétaire alors que les femmes s'occupent de l'agriculture vivrière (Rushigira, 2017).

L'analyse des résultats indique que le niveau d'adoption des pratiques de la GIR en production maraîchère dans la vallée du Niger au Bénin est relativement faible à cause d'un certain nombre de contraintes. Les pratiques de lutte culturale sont les méthodes les plus utilisées par les producteurs enquêtés pour la GIR. Cela pourrait s'expliquer par le fait que ces pratiques sont en général endogènes et connues par tous les producteurs et ne nécessitent pas de formation particulière. Quant aux pratiques biologiques et physiques 
(ou mécaniques), elles sont faiblement utilisées par les maraîchers pour le contrôle des bioagresseurs. Ce résultat serait dû au fait que ces pratiques nécessitent une certaine formation et assez de travail. Pour ce qui concerne les biopesticides et les extraits aqueux de plantes, les producteurs qui ne les adoptent pas estiment qu'ils sont inefficaces contre les ravageurs, chers et indisponibles. Un constat similaire a été fait par Adetonah et al. (2011) au Bénin et au Ghana qui ont estimé que le faible taux d'adoption des extraits aqueux de feuilles de neem ou de papayer est lié à une non maîtrise du processus de fabrication, à la pénibilité de la fabrication et à l'indisponibilité de la matière première en saison sèche (Adekambi et al., 2010 ; Adetonah et al., 2011). Les variétés résistantes ou tolérantes sont fortement adoptées parce que les producteurs estiment qu'elles permettent de réduire les attaques parasitaires. Des résultats similaires ont été observés par Nambie (2018) au niveau des maraîchers de Ouagadougou au Burkina-Faso.

Les facteurs déterminant l'adoption d'une innovation introduite en milieu rural sont liés non seulement à la technologie mais aussi aux conditions socioéconomiques et démographiques du producteur (Yabi et al., 2016). Dans la présente étude, cette influence varie d'une technologie à l'autre et selon le contexte. Ainsi, de l'analyse des résultats, il ressort que les maraîchers qui ont de vastes champs adoptent moins les pratiques de lutte biologique. L'étendue de la superficie emblavée constitue donc un frein à l'utilisation des pratiques de lutte biologique de la GIR en maraîchage dans la zone d'étude. Pour ce qui est de l'influence négative de l'expérience du maraîcher sur l'adoption des pratiques de lutte biologique de la GIR, cela peut sous-entendre que du fait de l'avantage comparatif coût bénéfice, les maraîchers sont parfois pressés des effets immédiats de l'application des extraits des feuilles de neem ou de papayer, et de ce fait, les producteurs seront réticents de les utiliser. Ce résultat corrobore les travaux de Feder (1982) et de Yabi et al. (2016) qui ont trouvé que le nombre d'années d'expérience du producteur influence négativement l'adoption d'une technologie ; et surtout que l'expérience étant un proxy de l'âge, les producteurs moins expérimentés seraient jeunes et plus disposés à prendre de risques plus élevés que les producteurs plus âgés. Par contre, le nombre d'actifs agricoles favorise l'adoption des pratiques de lutte biologique. Le nombre d'actif constitue donc une variable clé dans l'adoption des pratiques de lutte biologique. Ce résultat infirme les conclusions de Nambie (2018) qui a trouvé que le nombre d'actif n'a pas d'effet significatif sur la probabilité du choix de la stratégie des extraits aqueux de neem. Aussi, sont beaucoup plus disposés les maraîchers instruits à adopter les pratiques de lutte biologique. Ces résultats infirment ceux de Nambie (2018) qui stipulent que les maraîchers instruits de la ville de Ouagadougou au Burkina-Faso n'adoptent pas les extraits aqueux de neem comparativement à ceux non instruits. Selon Azontondé (2004), l'éducation est un facteur affectant 
l'adoption et l'application des innovations technologiques en milieu rural. En effet, plus les producteurs sont instruits, plus ils sont ouverts aux innovations technologiques. Le niveau d'instruction accroît donc le sens de l'innovation, l'habileté et la facilité d'apprécier les nouvelles technologies (Dakin, 2008). Une meilleure compréhension et l'esprit d'ouverture des lettrés aux innovations expliquent également nos résultats qui confirment ceux de Houeyissan (2006) sur les déterminants du choix et la volonté des riziculteurs du département des Collines à payer les semences des variétés améliorées mais ne corroborent pas ceux de Dakin (2008) qui est arrivé à la conclusion selon laquelle le niveau d'instruction affecte négativement l'adoption de des technologies vulgarisées par le Programme de Développement des plantes à Racines et Tubercules (PDRT). Les résultats de l'étude montrent également que face à la résistance des ravageurs, les maraîchers enquêtés sont réticents à l'utilisation des pratiques de lutte biologique de GIR. Par ailleurs, les différentes informations partagées par les ONG ou projets incitent les maraîchers à adopter les pratiques de lutte biologique de la GIR.

La pratique culturale constitue une innovation adoptée par les maraîchers expérimentés qui emblavent de grandes superficies et disposent d'un nombre important d'actifs agricoles. Il faut noter que cette pratique de la GIR est adoptée par les producteurs qui font face à des ravageurs résistants. De même, nos résultats indiquent que le sexe et le niveau d'instruction des producteurs contribuent énormément à rehausser le taux d'adoption des pratiques culturales de la GIR. Ces résultats confirment ceux observés par Issoufou et al. (2017) et ceux de Nambi (2018) qui ont rapporté que le niveau d'instruction influence positivement l'adoption des semences améliorées, des variétés résistantes ou tolérantes et la rotation culturale. Aussi, les maraîchers qui adoptent cette pratique de lutte appartiennent à des groupements. Ces cadres d'échange permettent aux maraîchers l'acquisition d'informations et de partage d'expériences, ce qui réduit leur incertitude et les aide à percevoir de manière plus objective l'innovation et les risques encourus (Kuhfuss et al., 2013; Parcell et Gedikoglu, 2013).

Quant aux pratiques de lutte physique, l'analyse des résultats révèle que les hommes qui produisent sur des superficies réduites, sont plus disposés à adopter ces pratiques de la GIR. Ces résultats rejoignent ceux obtenus par Namie (2018) chez les maraîchers au Burkina Faso qui stipulent que les hommes sont plus disposés à adopter toutes les pratiques la GIR excepté la rotation culturale, l'arrachage et la destruction des plantes malades qui sont beaucoup plus adoptées par les femmes en culture de chou. Cependant, les résultats de la présente étude sont en contradiction avec ceux de Sodjinou et al. (2015) qui ont montré que les femmes sont plus favorables à la production biologique que les hommes. 
Il est important de préciser que le contact avec un agent de vulgarisation n'a pas d'effet significatif sur l'adoption des différentes pratiques de la GIR considérées. Ce résultat pourrait s'expliquer par une insuffisance de l'appui-conseil ou de la formation de la part des agents en gestion intégrée des nuisibles à l'endroit des maraîchers. L'impact positif de la vulgarisation sur l'adoption des technologies agricoles a été révélé par plusieurs auteurs (Issoufou et al., 2017 ; Yabi et al., 2016 et Roussy et al., 2015). Les producteurs, qui sont en contact avec les agents de vulgarisation, bénéficient d'un encadrement plus rapproché. Ils accèdent donc facilement à l'information et à la formation sur les nouvelles technologies (Issoufou et al., 2017).

Dans l'optique de réduire et/ou d'encadrer l'utilisation des pesticides de synthèse et d'accroître le taux d'adoption des pratiques de gestion intégrée des bioagresseurs en production maraîchère au Bénin, s'avère nécessaire pour le gouvernement de promouvoir l'utilisation des pratiques de gestion intégrée des nuisibles en formant et en sensibilisant davantage les producteurs à travers des films documentaires, des champs écoles paysan, des parcelles de démonstrations et la vulgarisation.

\section{Conclusion}

La présente étude a été menée dans la vallée du Niger au Nord-Bénin, une des grandes zones productrices des cultures maraîchères. Conduite auprès de 280 producteurs, elle avait pour objectif d'identifier les facteurs susceptibles d'influencer l'adoption des pratiques de GIR dans la production. Des résultats, il ressort que des pratiques de GIR identifiées, ce sont les luttes culturales qui sont les plus adoptées.

L'étude a aussi montré que les facteurs déterminants l'adoption des pratiques de la GIR varient d'une pratique de lutte à une autre. La plupart de ces facteurs sont socio-économiques et démographiques et sont non seulement liés aux producteurs mais aussi à la pratique qu'ils utilisent. Ces résultats permettent de tirer plusieurs implications en termes de politiques agricoles pour l'amélioration de la qualité des produits maraîchers mais aussi la durabilité du maraîchage au profit de la sécurité alimentaire et de réduction de la pauvreté. Ainsi, le devenir de ces politiques dépendrait de comment les déterminants de l'adoption de la GIR identifiés par l'étude seront pris en compte. A ce titre, les chercheurs et les développeurs engagés dans la diffusion des pratiques de la GIR doivent renforcer les actions de l'information, la formation et la sensibilisation des maraîchers. 


\section{References:}

1. Adégbola P, Adékambi SA. 2008. Taux et déterminants de l'adoption des variétés améliorées d'igname développées par l'IITA. Rapport d'étape, $31 \mathrm{p}$.

2. Adékambi AS, Adégbola YP, Arouna A. 2010. Farmers' perception and agricultural technology adoption. The case of botanical extracts and biopesticides in vegetable production in Benin. Contributed Paper presented at the Joint 3rd African Association of Agricultural Economists (AAAE) and 48th Agricultural Economists Association of South Africa (AEASA) Conference, Cape Town, South Africa, September 19-23, 2010.

3. Adétonah S, Koffi-Tessio E, Coulibaly O, Sessou E, Mensah A G. 2011. Perceptions et adoption des méthodes alternatives de lutte contre les insectes des cultures maraîchères en zone urbaine et périurbaine au Bénin et au Ghana. Bulletin de la Recherche Agronomique du Bénin Numéro 69.

4. Amoussougbo, Y. 1993. Enquête sur l'usage des pesticides en cultures maraîchères dans la lutte contre les nématodes à galle. Thèse d'Ingénieur Agronome. FSA/UNB, 134p.

5. Assogba-Komlan F, Yarou BB, Mensah A, Simon S, 2012. Les légumes traditionnels dans la lutte contre les bioagresseurs des cultures maraîchères : associations culturales avec le Tchayo (Ocimumgratissimum) et le Yantoto (Launaeataraxacifolia). Fiche technique. Cotonou, Bénin : INRAB

6. Azontondé R. 2004. Impact économique de l'adoption des pratiques de la gestion intégrée de la fertilité des sols (GIFS) au Sud-Bénin : Cas d'Aplahoué (commune de Klouekanme) et de Banigbé (commune d'Ifangni). Thèse d'ingénieur agronome, Faculté des Sciences Agronomiques, Université d'Abomey- Calavi, Bénin, p.154.

7. Biaou D, Yabi J, Yegbemey R, Biaou G. 2016. Performances technique et économique des pratiques culturales de gestion et de conservation de la fertilité des sols en production maraîchère dans la commune de Malanville, Nord Bénin. International Journal of Innovation and Scientific Research, 21, 2351-8014.

8. Bourgeault J. 2009. Facteurs d'adoption de la lutte intégrée dans le secteur maraîcher en Montérégie (Québec). Mémoire de Maîtrise en Sciences de l'Environnement. Université du Québec/Montréal. Canada. $167 \mathrm{p}$.

9. CIMMYT, ECONOMICS PROGRAM. 1993. The adoption of agricultural technology: A guide for survey design. Mexico, D.F.: CIMMYT. 88 pp 
10. CIRAD-GRET. 2002. Ministère des Affaires étrangères, Mémento de l'Agronome, 1691pages.

11. Cramer JS. 1991. The Logit Model for Economists. London and New York: Edward Arnold; 154 pp.

12. Dagnelie P. 2006. Statistique théorique et appliquée: 2. Inférence statistique à 1 et 2 dimensions. Volume 2 de Statistique théorique et appliquée. Édition2 Éditeur De Boeck Supérieur, 2006 ISBN2804152294, 9782804152291 736pp. Diogo, R. V. C., E. M. M. Agandan, G. S.

13. Dakin L. 2008. Impact socio-économique des interventions du Programme de Développement des plantes à Racines et Tubercules (PDRT) sur les femmes rurales du département de la Donga. Thèse d'ingénieur agronome, Faculté d'Agronomie Université de Parakou, Bénin, p.106.

14. Fanou A, Glitho M, Baïmey H, Sagbohan J. 2004. Etude comparée des pesticides botaniques sur les organismes nuisibles des cultures maraîchères (carotte, oignon et gboma) dans les centres maraîchers d'Akron, de Sokomey, de Grand-popo et d'Adjohoun. In Actes de l'Atelier Scientifique National, 289-306.

15. FAO, Organisation des Nations Unies pour l'Alimentation et l'Agriculture. 2011. La pratique de la gestion durable des terres. Directives et bonnes pratiques pour l'Afrique subsaharienne: Application sur le terrain. TerrAfrica, WOCAT, Rome, Italie.241p.

16. Feder G. 1982. Adoption of interrelated agricultural innovations complementary and the risk, scale and credit. American Journal of Agricultural Economics, 12: 59-73.

17. Hasan SMM. 2017. Factors Affecting Integrated Pest Management (IPM) Adoption and Pesticide Use in Kenyan Vegetable Farmers. Thesis submitted to the faculty of Virginia Tech University in partial fulfillment of the requirements for the degree of Masters in Science In Agricultural and Applied Economics, 106p.

18. Houeyissan S. 2006. Déterminants des choix et de la volonté de payer les semences des variétés améliorées de riz dans le Département des Collines, Thèse d'ingénieur agronome, Faculté des Sciences Agronomiques, Université d'Abomey- Calavi, Bénin, P.94

19. Houndété TA, Kétoh GK, Hema OSA, Brévault T, Glitho IA, MartinT. 2010. Insecticide resistance in field populations of Bemisia tabaci (Hemiptera: Aleyrodidae) inWest Africa. Pest Management Science 66: 1181-1185. DOI:10.1002/ps.2008.

20. Hurlin C. 2003. Econométrie des variables qualitatives. Cours de maîtrise d'économétrie, France Université d'Orléans, 59 p. 
21. Issoufou OH, Boubacar S, Adam T, Yamba B. 2017. Déterminants de l'adoption et impact des variétés améliorées sur la productivité du mil au Niger. African Crop Science Journal, Vol. 25, No. 2, pp. 207 - 220.

22. James B, Godonou I, Baimey H, Goergen G, Toko RSM. 2010. Gestion intégrée des nuisibles en production maraîchère : Guide pour les agents de vulgarisation en Afrique de 1 ' Ouest. Institut international d'agriculture tropicale (IITA), Ibadan, Nigeria, 120 p.

23. Kuhfuss L, Préget R, Thoyer S. 2013. Préférences individuelles et incitations collectives: quels contrats agro-environnementaux pour la réduction des herbicides par les viticulteurs. Revue d'Études en Agriculture et Environnement/Review of Agricultural and Environmental Studies 95: 111-143.

24. Maddala GS. 1983. Limited-Dependent and qualitative variables in Econometrics. Cambridge : Cambridge University Press.

25. Nabie B. 2018. Analyse des pratiques phytosanitaires et des facteurs d'adoption de la gestion intégrée des nuisibles en production maraîchère en milieu urbain et périurbain au Burkina Faso : Cas de la ville de Ouagadougou. Travail de fin d'étude du Master de spécialisation en production intégrée et préservation des ressources naturelles en milieu urbain et péri-urbain, Gembloux Agro-Bio Tech. Université de Liège. Belgique, 96p.

26. Odjouola IAM. 2017. Déterminants de l'utilisation à long terme des extraits botaniques et/ou biopesticides en agriculture urbaine et périurbaine sur les sites maraîchers au Bénin. Travail de fin d'étude du Master de spécialisation en production intégrée et préservation des ressources naturelles en milieu urbaine et périurbaine, Gembloux Agro-Bio Tech. Université de Liège. Belgique, 59p.

27. Parcell JL, Gedikoglu H. 2013. A Differential Game Approach to Adoption of Conservation Practices. 2013 Annual Meeting, August 46, 2013, Washington, DC, Agricultural and Applied Economics Association, 14p.

28. Parsa S, Morseb S, Bonifacioc A, Chancellord CBT, Condorie B, Crespo-Pérezf V, Hobbsg LAS, Kroschelh J, Bai NM, Rebaudoj F, Sherwoodl GS, Vanekm SJ, Fayej E, Herreraf AM, Dangles O. 2014. Obstacles to integrated pest management adoption in developing countries. PNAS, vol. 111. no. 10.3889-3894.

29. Petit S. 2015. Faut-il absolument innover ? À la recherche d'une agriculture d'avant-garde. Courrier de l'environnement de l'Inra n ${ }^{\circ} 65$. 19-28.

30. Rasmussen S. 2011. The Basic Theory of Production Optimisation. Springer Heidelberg Dordrecht London New York. ISBN 978-3-64214609-1. 287 p. 
31. Roussy C, Ridier A, Chaib K. 2015. Adoption d'innovations par les agriculteurs : rôle des perceptions et des préférences. Working Paper SMART-LERECO $\mathrm{N}^{\circ} 15-03$. https://ageconsearch.umn.edu/bitstream/206036/2/WP15-03.pdf. Consulté le 25 mars 2019.

32. Rushiguira C. 2017. Analyse technico-économique de la production maraîchère à Kamanyola dans la plaine de Ruzizi/RDC. Travail de fin d'étude du Master de spécialisation en développement, environnement et sociétés. Faculté des sciences économique, sociale, politique et de communication, Université Catholique de Louvaine, Belgique, 129p.

33. Sale A, Folefack PD, Obwoyere GO, Lenah Wati N, Lendzemo WV, Wakponou A. 2014. Changements climatiques et déterminants d'adoption de la fumure organique dans la région semi-aride de Kibwezi au Kenya. Int. J. Biol. Chem. Sci. 8(2): 680-694. DOI : http://dx.doi.org/10.4314/ijbcs.v8i2.24

34. Sodjinou E, Glin LC, Nicolay G, Tovignan S, Hinvi J. (2015). Socioeconomic determinants of organic cotton adoption in Benin, West Africa, Agric. Food Econ., vol. 3, no 12, p. 22, 2015.

35. Son D, Zerbo KBF, Bonzi S, Legreve A, Somda I, Schiffers B, 2018. Assessment of Tomato (Solanumlycopersicum L.) Producers' Exposure Level to Pesticides, in Kouka and Toussiana (Burkina Faso). Int. J. Environ. Res. Public Health 2018, 15, 204

36. Tiamyhou B, Sodjinou E. 2003. Etude diagnostic de la filière maraîchage dans la vallée du Niger. PADSE.

37. Yabi AJ, Bachabi XF, Labiyi AI, Ode AC, Ayena LR. 2016. Déterminants socioéconomiques de l'adoption des pratiques culturales de gestion de la fertilité des sols utilisées dans la commune de Ouaké au Nord-Ouest du Bénin. Int. J. Biol. Chem. Sci. 10(2): 779-79

38. Zongo B, Diarra A, Barbier B, Zorom M, Yacouba H, Dogot T. 2015. Farmers' practices and willingness to adopt supplemental irrigation in Burkina Faso. International Journal of Food and Agricultural Economics ISSN 2147-8988 Vol. 3 No. 1, pp. 101-117 